Working Paper no. 59

\title{
Empirical modeling of deprivation contagion among social exclusion dimensions (using MCMC methods). §
}

\author{
Ambra Poggi \\ LABORatorio R. Revelli, Centre for Employment Studies
}

Xavier Ramos

Universitat Autònoma de Barcelona and IZA

This version: February 2007

Laboratorio R. Revelli, Collegio Carlo Alberto

Via Real Collegio, 30 - 10024 Moncalieri (TO)
Tel. +39011670.50.60 - Fax +39011670.50.61 www.laboratoriorevelli.it - labor@laboratoriorevelli.it

LABOR is an independent research centre of the Collegio Carlo Alberto

$\S$ Financial support from the Spanish Ministerio de Educacion y Ciencia (Ref: SEJ 2004-07373-C03-01/ECON) is gratefully acknowledged. 


\title{
Empirical modeling of deprivation contagion among social exclusion dimensions (using MCMC methods). s
}

\author{
Ambra Poggi \\ Collegio C. Alberto, LABORatorio R. Revelli - Centre for Employment Studies \\ Xavier Ramos \\ Universitat Autònoma de Barcelona and IZA
}

This version: February 2007

\begin{abstract}
Economic theory and empirical evidence clearly show that social exclusion dimensions are inter-related. Notwithstanding that, dimensions are usually assumed independent from one another in the economics literature. In this paper we explore the inter-dependency of social exclusion dimensions and study the transmission of deprivation among them. In particular, we propose the use of stochastic epidemic models, which are typically used to study the transmission of infectious diseases, to the analysis of deprivation diffusion among social exclusion dimensions with the aim of acquiring a deeper understanding of the mechanism governing deprivation transmission. We also provide an empirical implementation that investigates the consequences, in terms of future deprivation, for Italian and Spanish women of being jobless, as opposed to doing paid work. We also investigate the consequences of being unemployed versus being inactive. We conclude that working seems to act as a protective mechanism to shocks. In addition, conditional on losing one's job, women who subsequently search for a job (unemployed) are more likely to experience contagion than women who do not search (inactive).
\end{abstract}

Keywords: deprivation, social exclusion, contagion models, Markov chain Monte Carlo methods, metropolis-Hastings algorithm, stochastic models, propensity score.

JEL Classification: C11, C15, I30

$\S$ Financial support from the Spanish Ministerio de Educacion y Ciencia (Ref: SEJ2004-07373-C03-01/ECON) is gratefully acknowledged. 


\section{Introduction}

Over recent years, and more evidently since the EU Lisbon summit in 2000 , social exclusion -or better, social inclusion - has become an increasingly important issue on the agenda of many European institutions, being also very present in many policy debates in Europe and elsewhere. This has triggered a renewed interest in the topic from both institutional and academic spheres ${ }^{1}$. We have seen, for instance, considerable advances in its measurement (Bossert, D'Ambrosio and Peragine, 2005; D'ambrosio and Chakravarty, 2003), in the empirical identification of the socially excluded (Whelan et al., 2002; Tsakloglou and Papadopoulos, 2002), or in the study of the dynamics of social exclusion (Poggi, 2007a and 2007b). However, our understanding of other relevant aspects of social exclusion is still rather limited. This paper deals with one of these aspects that, to the best of our knowledge, has never captured the attention of economist and social scientists in general; namely, the transmission of deprivation among social exclusion dimensions.

Two distinctive and central features of the notion of social exclusion are its relativity and multi-dimensionality (Atkinson, 1998; Sen, 2000). ${ }^{2}$ Relativity stems from the impossibility of understanding exclusion without context or reference, which is normally taken to be the society where the individual belongs. The multidimensionality embraces economic, social and political aspects, and it has induced many social scientists to approach the concept from Sen's capability approach (1985), as the failure to attain adequate levels of functionings that are deemed valuable in their society (i.e. Sen, 2000; Bossert, D’Ambrosio, Peragine, 2005; Burchardt et al. 2002; Poggi 2007a and 2007b).

Economic theory and empirical evidence clearly show that these dimensions are inter-related, i.e. they are not independent from each other. For instance, lower levels of education and poor housing conditions are correlated with poorer average health (references), and poor health is correlated with lower education attainment (references). Notwithstanding that, dimensions are usually assumed independent from one another in the economics literature. In this paper we explore the inter-dependency of social exclusion dimensions and study the transmission of deprivation among them. In particular, we propose the use of stochastic epidemic models (Andersson and Britton, 2000), which are typically used to study the transmission of infectious diseases, to the analysis of deprivation diffusion among social exclusion dimensions with the aim of acquiring a deeper understanding of the mechanism governing deprivation transmission.

The empirical part of the paper investigates the consequences, in terms of future deprivation, for women of being jobless, as opposed to doing paid work. There is ample evidence that women with children often decide not to work and look after their offspring (e.g. Ahn and Mira, 2002, Jaumotte, 2003). However, the decision not to work may lead to (higher chances of) future exclusion, due for instance to faster transmission of deprivation from one dimension to the others or to simply because they are more vulnerable to negative shocks. This is clearly an empirical question and we shall address it by means of our stochastic epidemic model and using data from the ECHP for the period 1994-2001.

This paper contributes to the literature in three ways. First, we propose a methodology to analyze deprivation transmission, which permits investigate the interdependency existing across social exclusion dimensions. Second, we help answer a very important empirical question: which are the consequences in term of deprivation transmission for women of being jobless, as opposed to doing paid work? Note that the answer to this question may have important policy implications. Third, we offer new empirical evidence on deprivation and social exclusion conditions in Italy and Spain.

The remainder of the paper proceeds as follows. The next section is concerned about the modeling of the transmission of deprivation across social exclusion dimensions; estimation and inference issues are the subject matter of section 3, while section 4 observes manners of dealing with heterogeneous social exclusion dimensions and populations. Our empirical application to jobless women provides a relevant illustration of the methodology put forth in the previous sections. Finally, section 6 concludes with avenues for further research.

\footnotetext{
${ }^{1}$ The institutional response to the Lisbon summit agreements was an unprecedented 70 million euro programme launched by the European Commission to promote social inclusion - with one of the main aims being to improve our understanding of social exclusion.

${ }^{2}$ Other relevant features include agency and dynamics. Notwithstanding that, these are not so much a concern of this paper.
} 


\section{Modelling deprivation transmission among social exclusion dimensions}

Social exclusion comprises several dimensions, which are assumed interdependent. Deprivation in a given dimension exists if the individual fails to attain adequate levels of achievement in that dimension - how exactly deprivation is to be measured is an empirical issue that will be tackled below in our empirical illustration. A dimension is defined susceptible if it is not deprived, i.e. if the individual does not suffer deprivation in that dimension. Two sources of infection are considered. Susceptible dimensions may be infected by a given infective dimension or by a factor external to the individual (e.g. job loss, divorce, sickness), and once infected they become infective. Thus, to remain susceptible at time $t+1$ a dimension must escape infection by the external source of infection and must escape infection from any other infected dimension of the individual. We want to model the transmission or infection of deprivation among dimensions from time $t$ to $t+1$.

Let $q_{e}$ denote the probability that a susceptible dimension at time $t$ escapes external infection at $t+1$, and let $q_{n}$ denote the probability that a susceptible dimension at $t$ escapes infection at $t+1$ when exposed to one infected dimension. Then, the probability that exactly $j$ of the $s$ initial susceptible dimensions of a given individual are infected from $t$ to $t+1, w_{j s}$, is given as (Longini and Koopman, 1982)

$$
\omega_{j s}=\left(\begin{array}{c}
s \\
j
\end{array}\right) \omega_{j j}\left(q_{e} q_{n}^{j}\right)^{s-j}
$$

where

$$
\omega_{j j}=1-\sum_{i=0}^{j-1} \omega_{i j}
$$

Lefèvre and Picard (1990) show that a closed form for $w_{j s}$ can be obtained in terms of a non-standard family of polynomials called Gontcharoff polynomials. Let $U$ be a sequence of real numbers $u_{0}, u_{1}, \ldots$ Then, the Gontcharoff polynomials associated with $U$ are defined recursively by

$$
\begin{gathered}
G_{0}(x \mid U)=1, \\
G_{j}(x \mid U)=\frac{x^{j}}{j !}-\sum_{i=0}^{j-1} \frac{u_{i}^{j-i}}{(j-i) !} G_{i}(x \mid U) \quad(j=1,2, \ldots) .
\end{gathered}
$$

Following O'Neill et al. (2000), define $H_{j}=\omega_{j j} / j$ !. Using (1) and (2), we obtain

$$
H_{j}=\frac{1}{j !}-\sum_{i=0}^{j-1} \frac{1}{(j-i) !}\left(q_{e} q_{n}^{i}\right)^{j-i} H_{i}
$$

By comparison with equations (3) it follows that $H_{j}=G_{j}(1 \mid U)$, where $U$ is the sequence with ith term $u_{i}=q_{e} q_{n}^{i}$. Finally, the closed form for $w_{j s}$ can be written as

$$
\omega_{j s}=\left(\begin{array}{c}
s \\
j
\end{array}\right)\left(q_{e} q_{n}^{j}\right)^{s-j} j ! G(1 \mid U) .
$$

It is important to bear in mind that dimensions are assumed homogeneous in two relevant aspects: they do not differ in their potential to infect others (e.g. housing deprivation has the same potential as health deprivation to infect, say, the socializing dimension), and all dimensions have the same probability of being immune to deprivation. Below we discuss ways to relax these simplifying restrictions. 


\section{Estimation Method and Inference: Markov chain Monte Carlo}

We use bayesian inference and Markov chain Monte Carlo (MCMC) methods to estimate the two parameters of interest, $q_{e}$ and $q_{n}$, required to obtain an estimate of the probability that exactly $j$ of the $s$ initial susceptible dimensions of a given individual are infected from $t$ to $t+1, w_{j s}$.

For this we need to compute the joint posterior of $q_{e}$ and $q_{n}, \pi\left(q_{e}, q_{n} \mid n_{j s}\right)$, where $n_{j s}$ denotes the number of individuals who had $s$ a priori susceptible dimensions at time $t$ and ended up with $j$ infected dimensions at $t+1$. Assuming initial independence between the two escaping (external and internal) infection probabilities, $q_{e}$ and $q_{n}$, from Bayes' theorem we have

$$
\pi\left(q_{e}, q_{n} \mid n_{j s}\right) \propto L\left(q_{e}, q_{n}\right) \pi\left(q_{e}\right) \pi\left(q_{n}\right),
$$

where $\pi\left(q_{e}\right)$ and $\pi\left(q_{n}\right)$ are the prior distribution of $q_{e}$ and $q_{n}$, and $L\left(q_{e}, q_{n}\right)$ the likelihood function, is given by

$$
L\left(q_{e}, q_{n}\right)=\prod_{s=1}^{S} \prod_{j=0}^{s}\left(\omega_{j s}\right)^{n_{j s}}
$$

which can be computed by using equation (4). We use a particular method of MCMC known as Metropolis-Hastings algorithm (see Gilks et al., 1996) to sample from the joint posterior distribution. The algorithm works as follows. Suppose a likelihood $L$ and a prior density $\pi$. By Bayes's theorem, the posterior density is $c L \pi$, where $c$ is the normalising constant. Now, construct a Markov chain $\left\{Z_{n}\right\}$ as follows: given the current state of the chain, say $Z_{n}=x$, draw a possible new (candidate) point, $y$, from a proposal density $q(y \mid x)$. Accept the candidate with probability

$$
\min \left\{1, \frac{L(y) \pi(y) q(x \mid y)}{L(x) \pi(x) q(y \mid x)}\right\},
$$

in which case $Z_{n+1}=y$. If the candidate is rejected, then set $Z_{n+1}=x$. Under mild conditions, the stationary distribution of this Markov chain is the posterior density (Gilks et al., 1996). Thus, to sample from the joint posterior distribution we simply run the Markov chain until it is deemed to have converged and then draw samples from the output.

Since our two parameters have range $(0,1)$, we transform each one by using the logistic transformation

$$
t \rightarrow \tilde{t}=\log \frac{t}{(1-t)}
$$

so that the transformed parameters all have range $\mathrm{R}$, and a normal proposal density can be used. The convenience of using such proposal density is that the acceptance probability of (6) reduces to

$$
\min \left\{1, \frac{L(y) \pi(y)}{L(x) \pi(x)}\right\} .
$$

In order to compute the joint posterior of $q_{e}$ and $q_{n}$, notice that

$$
t=\frac{\exp (\tilde{t})}{(1+\exp (\tilde{t}))}=g(\tilde{t})
$$

and that the Jacobian of this transformation is given by

$$
J(\tilde{t})=\frac{\exp (\tilde{t})}{(1+\exp (\tilde{t}))^{2}} .
$$


Then, the joint posterior we actually compute is

$$
\pi\left(q_{e}, q_{n} \mid n_{j s}\right) \propto L\left(g\left(\widetilde{q}_{e}\right), g\left(\widetilde{q}_{n}\right)\right) \pi\left(g\left(\widetilde{q}_{e}\right)\right) \pi\left(g\left(\widetilde{q}_{n}\right)\right) J\left(g\left(\widetilde{q}_{e}\right)\right) J\left(g\left(\widetilde{q}_{n}\right)\right) .
$$

\section{Modelling Heterogeneity}

In Section 2, we have assumed that both the population and dimensions are homogenous. We now discuss how to relax these simplifying assumptions, starting with the latter. As mentioned above, assuming homogeneous dimensions means that they do not differ in their potential to infect others, and that all dimensions have the same probability of being immune to deprivation. Therefore, we can incorporate heterogeneity in the model in the following two ways.

First, we allow dimensions to differ in their potential to infect others, by letting $q_{n}$ depend on the deprivation intensity. ${ }^{3}$ We let $q_{n}=\Phi_{Y}(\lambda)$, where $\Phi$ is the Poisson probability distribution, $\lambda$ is the arrival rate (for hypothesis equal to one) and $Y$ indicates the intensity of deprivation. ${ }^{4}$ The closed form for $w_{j s}$ is analogous to the one derived by Ball et al. (1997) considering a time-driven process (where $Y$ indicates the length of the infectious period), and it may be written as:

$$
\omega_{j s}=\frac{1}{(s-j) !} \sum_{i=s-j}^{s} \frac{s !}{(s-i) !} \phi_{Y}(i)^{s-i} q_{e}^{i} G_{i-s+j}\left(0 \mid E^{s-j} U\right)
$$

where $U$ is the sequence with ith term $u_{i}=\phi_{Y}(i)$ and $E^{i} U$ denotes the sequence $u_{i}, u_{i+1}, \ldots$.

Second, suppose that each susceptible dimension has some probability $v$ of being immune to deprivation, perhaps due to (inherited) wealth (for housing deprivation), as a result of their cautious and healthy behaviour (for health deprivation), or of their education level (for monetary poverty). In other words, $v$ can be defined as the probability that a certain dimension corresponding to a certain individual is protected from deprivation (if $v=0$ we assume homogeneity). Thus, the number of unprotected susceptible dimensions available at the start of the contagious process has a binomial distribution and the closed form for $w_{j s}$ can be written as (O'Neill et al., 2000):

$$
\omega_{j s}(v)=\sum_{i=0}^{s-j}\left(\begin{array}{l}
s \\
i
\end{array}\right) v^{i}(1-v)^{s-i} \omega_{j s-i}
$$

Let us now turn to explicitly model population heterogeneity. Should we be interested in analysing transmission probabilities by population subgroups, say gender, we could partition the population into two mutually exclusive groups $(k=1,2)$, and simply write the closed form for $w_{j s}$ as:

$$
\omega_{j s}=\left(\begin{array}{c}
s \\
j
\end{array}\right)\left(\left(q_{e}+d_{e}\right)\left(q_{n}+d_{n}\right)^{j}\right)^{s-j} j ! G(1 \mid U) .
$$

where $d_{e}$ and $d_{n}$ are assumed zero if $\mathrm{k}=2$ (with $\left(q_{e}+d_{e}\right) \in[0,1]$ and $\left(q_{n}+d_{n}\right) \in[0,1]$ if $\left.\mathrm{k}=1\right)$. Note that the above expression can be easily extended to the case of $n$ groups.

However, as is well known, as long as other attributes are also responsible for the outcome of interest, this procedure will not be able to identify the true effect of interest (e.g. the effect of a particular characteristic or of a policy) because it will be confounded by the influence of all these other attributes. In other words, the effect of the treatment may be confounded with that of the factors that lead individuals to select different treatments. One means for adjusting

\footnotetext{
${ }^{3}$ Note that we are also introducing some degree of individual heterogeneity since deprivation intensity in dimension $j$ may differ across individuals.

${ }^{4}$ The considered Poisson process is not a time-driven process: it is an event-driven process. It evolves only if a different degree of deprivation is reached. Note that deprivation intensity can be empirically computed using gap indices when data are available.
} 
between-group comparisons for pre-existing differences involves statistical matching. ${ }^{5}$ These methods generally specify a function measuring the proximity of one individual of the treatment group to one not-treated individual based on many covariates. This way, it is possible to create a comparison group for the treatment group minimizing the distance between matched cases. To do so, recent research has explored the use of the propensity score - that is, the predicted probability that an individual receives the treatment of interest. The propensity score captures all the variance in the covariates relevant for adjusting between-group comparisons (Rosenbaum and Rubin, 1983); as a result, one can simply match two groups based on this single variable. Once the two groups have been matched, it is possible to compute $q_{e}$, and $q_{n}$, for each group and compare the results across groups. This is the method we shall employ in our empirical application to study the effect of being jobless on contagion probabilities.

\section{Empirical application}

Joblessness is usually viewed as a bad thing, especially if it is involuntary. It is very much related to poverty (Nickell, 2004), increases dependency between family members (e.g. spouses and youth), brings frustration and unhappiness if the lack of work is not desired (Clark and Oswald, 1994; Di Tella, MacCulloch and Oswald, 2003), - which ought to include unemployed but also some inactive-, and it is also inefficient. However, we do not know much about the effects of having no work today on tomorrow's likelihood of experiencing deprivation, and about the mechanisms that govern such effects. Whether being jobless leads to future exclusion, due to faster transmission of deprivation from one dimension to the others, or simply because jobless people are more vulnerable to external shocks, is an empirical question. The purpose of this section is to find answers to these questions, and illustrate how our methodology is useful to provide helpful suggestion for policy design. To do so we analyse women in Italy and Spain, where they face low participation rates, high unemployment rates, and are often seen out of the labour force when close relatives need care.

Social exclusion can be defined as a process that fully or partially excludes individuals or groups from social, economic and cultural networks of the society they live in. As we stressed above, social exclusion is a multidimensional concept, but the issue of which are the dimensions deemed relevant to identify an individual as excluded (and how to select them) is subject to ongoing discussion since a complete list cannot be unequivocally compiled (see, among others, Poggi, 2007a and 2007b; Brandolini and D'Alessio, 1998). Following the guidance offered by the previous literature, we select five relevant dimensions to capture all the principal aspects of social exclusion. The selected dimensions are "having an adequate income", "basic needs fulfillment", "having an adequate house", "the ability to have social relationships" and "being healthy". The first three dimensions describe the economic features of social exclusion, and the remaining two dimensions emphasize the social features of exclusion.

We use all eight waves (1994-2001) of the European Community Household Panel (ECHP). The ECHP is a multicountry comparative household panel survey conducted annually by following the same sample of households in Member States of the European Union. The advantage of the ECHP is that it permits us to analyse economic and social household conditions from a dynamic point of view. However, a significant disadvantage is the omission of the homeless, who are most likely socially excluded. Table 1 summarizes the operationalization of the five dimensions of social exclusion: it shows the ECHP items selected to correspond to each dimension. Note that most of the items are household level variables while others are individual level ones (note also that some items refer to subjective individual evaluations). For each selected item, we assigned a score of zero if the individual can afford the item (has the item or does not have 'the problem'), ${ }^{6}$ and a score equal to one if the individual is deprived of that item. The individual is considered deprived in a given dimension if she suffers deprivation in at least one item.

The unit of analysis is the individual: in particular, Italian and Spanish women aged 16-65 years. As pointed out above, both Italy and Spain exhibit low female labour market participation rates and strong family networks. Moreover, both countries are particularly interesting cases for the study of deprivation transmission because, during the period of study, they exhibit high levels of unemployment, severe poverty, and the abrupt decline in the traditional form of family-based care (European Commission, 2001; OECD, 2005; Adiego and Moneo, 2004; Arriba and Moreno, 2002; Jonsson, 2003).. Hence, we believe that the comparison of the results across these two countries, as well as of the pooled data, makes sense and may lead to interesting conclusions.

\footnotetext{
${ }^{5}$ See Caliendo and Kopeining (2005) for a technical review.

${ }^{6}$ For example, she can afford a durable or she has an indoor flushing toilet or she has a very good/good/ fair health or she talks to (meets with) any of the neighbour at least once a month or she does not have an accommodation with damp walls.
} 
First we evaluate if deprivation transmission differs between women who continue working and women who are not working any longer. We initially select a sample of women that are working at year $t-1$, and from this, choose the subsample of women that become jobless at year $t$ (and remain jobless in $t+1$ ): this is our treatment group. The comparison group is the sub-sample of women that remain in paid work also in years $t$ and $t+1$. Were we to compute the probabilities of deprivation transmission and to compare the results between groups, we would confound the effect of changes in employment status with the influence of many other observable attributes which differ between treatment and comparison groups, and which are also responsible for deprivation transmission (see Appendix A). To solve this problem, we implement propensity score matching using a score computed from a logit model, which estimates the probability of becoming jobless conditional on a set of pre-treatment observable characteristics basically personal characteristics and job attributes measured at year $t$ (when all the women are working). In order to choose the specification of the logit model, we start with a parsimonious specification and then test up by iteratively adding variables to the specification until tests for mean differences in covariates between treatment and comparison units become statistically insignificant (see Appendix A). Note that the key identifying assumption in the propensity score matching technique is that the outcome be independent of the change in employment status, conditional on the observable characteristics. A region of common support is assumed. ${ }^{7}$ We use the caliper matching method, a refinement of the nearest neighbour method which allows matches within a specified distance of the score of the treatment unit, thus permitting to eliminate bad matches. Results of the logit models on becoming jobless are reported in Appendix A. Descriptive statistics of the treatment group of women by country are reported in Table 2 columns (a). About $23 \%$ of the women in the treatment group are poor, $22 \%$ do not achieve the basic needs fulfillment, about $20 \%$ suffer some housing deprivation, about $6 \%$ have some health problems, and $10 \%$ suffer social relationship deprivation. The susceptible dimensions are on average about 4.

Table 3 reports the estimates of our stochastic epidemic model for treatment and control women. Let us first look at the pooled sample including both Italian and Spanish data. The probability that a susceptible dimension at time $t$ escapes deprivation infection by external factors at $t+1\left(q_{e}\right)$ for jobless women and for control women is, respectively, 0.901 and 0.939 . In other words, deprivation contagion by external forces is $3.80 \%$ more likely for jobless women than for women in paid work. On the other hand, the probability that a susceptible dimension at time $t$ escapes deprivation infection at $t+1$ when exposed to one infected dimension $\left(q_{n}\right)$ for jobless women and for control women is, respectively, 0.984 and 0.987 . This means that deprivation transmission within dimensions (contagion due to internal forces) is slightly more likely for jobless women than for women in paid work $(0.30 \%)$. Note that we find similar results when performing the analysis for each country separately. This evidence suggests that working seems to act as a protective mechanism to shocks, being particularly effective against shocks due to external forces. By construction of our matching strategy, both groups should face similar ex-ante distributions of external and internal shocks; that is, provided we capture all relevant determinants of those distributions in our matching variables. Therefore, such prophylactic effect arises from the beneficial sides of working per se, be it higher earnings and thus income, better mental health or richer socializing.

The jobless women above are heterogeneous in one important aspect that might have a bearing on contagion probabilities: some are actively looking for a job whereas some others are not. That is, they are either inactive or unemployed. Next we investigate whether inactivity leads to different deprivation transmission probabilities than unemployment. To do so, we define a new treatment group of inactive women and a new control group of unemployed women, and use propensity score matching with the same covariates as before. Information about matching quality and results of the logit model on inactivity are reported in Appendix $B$, descriptive statistics of the composition of the treatment group (inactive women) are presented in column (b) Table 2, and deprivation escape probabilities for treatment and control groups are shown in Table 4. We find that deprivation contagion by external and internal forces are respectively 2.6 and 5.1 percentage points more likely for unemployed than for inactive women. Given that the two groups are observationally equivalent at $t-1$, due to our matching strategy, the differences in contagion probability estimates ought to be attributed to the direct effect of unemployment and inactivity. For instance, the difference in contagion probabilities may be a reflection of the differential effect of the sudden drop in income caused by the job loss, which in turn obliges some women to search for another job.

Finally we will apply our methodology to the analysis of women, whose inactivity is due to housework chores and duties, child caring and rearing or looking after other dependent persons. ${ }^{8}$ This is an especially relevant group since there is plenty of evidence that women with children often decide not to work and look after their offspring (e.g. Ahn and Mira, 2002). The interesting and policy relevant issue is whether these women are more or less exposed to

\footnotetext{
${ }^{7}$ We are aware that this procedure entails some potential problems: the support condition may fail in interior regions; good matches could be lost near the boundary of support region, and excluding observations in either group may change the parameter being estimated.

${ }^{8}$ They represent $55.6 \%$ of all inactive women in our sample, see Table 5.
} 
deprivation contagion or more or less vulnerable to external shocks than other inactive women relative to women with identical relevant characteristics who nevertheless decide to look for a job.

Estimates from the two matched samples ${ }^{9}$ show that deprivation contagion by external forces is slightly more likely for these inactive women than for unemployment women $(0.80 \%)$, while the opposite is true for internal contagion (Table 8). That is, deprivation contagion within dimensions is much more likely for unemployed women than for inactive ones $(6.9 \%)$. In order to understand these results, compare the probabilities of inactive and unemployed women of tables 4 and 6 . Contagion probabilities of inactive women do not change, despite referring to different groups of inactive women -which suggests that the type of inactivity may not be relevant. Therefore, the differences in internal and external contagion probabilities between inactive and unemployed women are mostly due to the change in probabilities between the two groups of unemployed women. As columns (b) and (c) of Table 2 show, these two groups do not have the same characteristics, and some of these differences may be responsible for the different contagion probabilities. In other words, those looking for a job in a situation where many decide to remain inactive are probably in need, and thus both external and internal shocks are more likely to hurt, as compared to all unemployed women.

\section{Concluding Remarks}

In this paper, we explore the inter-dependency of social exclusion dimensions and study the transmission of deprivation among them. In particular, we propose the use of stochastic epidemic models (typically used in epidemiological analysis but, as far as we know, never used before in economics) to study the deprivation diffusion among social exclusion dimensions with the aim of acquiring a deeper understanding of the mechanism governing deprivation transmission. We also show how Bayesian inference and Markov chain Monte Carlo methods can be employed to empirically estimate the parameters of interest, i.e. the probability of avoiding infection from factors external to the individual (e.g. job loss, divorce, sickness) and the escape probability of within dimension deprivation transmission. We begin assuming that population and dimensions are homogenous, and then discuss how to relax these simplifying assumptions.

The empirical section of the paper illustrates how our methodology can be employed to investigate policy relevant issues. In particular, we investigate the effects on internal and external deprivation contagion of not working, distinguishing the unemployment from the inactivity. To do so, we use the Italian and Spanish data included in the European Community Household Panel. In order to compare groups of women and identify the effect of the treatment (i.e. changes in employment status or the decision of being inactive), we implement propensity score matching.

Our findings suggest that working seems to act as a protective mechanism both to external shocks and, to a lesser extent, to internal contagion. That is, on top of the obvious effects on current financial situation, working procures better inclusion prospects for tomorrow. Conditional on losing one's job, women who subsequently search for a job are more likely to experience deprivation contagion than women who do not search. However, women whose inactivity is due to housework duties, child caring and rearing or looking after other dependent persons, face larger external contagion probabilities than observationally equivalent unemployed women.

\footnotetext{
${ }^{9}$ The treatment group consists now of women whose inactivity is due to housework or caring duties. See Appendix C for information on matching quality and logit estimates for propensity score.
} 


\section{References}

Adiego, M., Moneo, C.(2004), "Poverty and persistent poverty in Spain, 1994-2001", in: Social Indicators of Spain 2004, Instituto Nacional de Estadistica, Madrid.

Ahn, N. and P. Mira (2002) "A note of the relationship between fertility and female employment rates in developed countries", Journal of Population Economics, 15: 667-682.

Andersson, H. and T. Britton (2000), Stochastic Epidemic Models and Their Statistical Analysis, Lecture Notes in Statistics 151, New York: Springer.

Arriba, A., Moreno, L. (2002), "Spain: Poverty, social exclusion and safety nets", Documento de trabajo 02-10, Unidad de Politicas Comparadas (CISC).

Atkinson, A.B. (1998), "Social exclusion, poverty and unemployment", in A.B. Atkinson and J. Hills (eds.), Exclusion, Employment and Opportunity, CASE/4, Centre fro Analysis of Social Exclusion, London School of Economics, 1-20.

Bossert, W., C. D'Ambrosio and V. Peragine (2007), "Deprivation and Social Exclusion", Economica, forthcoming.

Burchardt, T., J. LeGrande and D. Piachaud (2002), "Degrees of exclusion: developing a dynamic multidimensional measure", in "Understanding social exclusion", edited by J. Hills, J. Le Grand and D. Pichaud

Caliendo, M. and S. Kopeinig (2007), "Some practical guidance for the implementation of propensity score matching", Journal of Economic Surveys, forthcoming.

Chakravarty, S. and C. D'ambrosio (2003), "The measurement of social exclusion”, DP DIW: Berlin.

Clark, A.E. and A.J. Oswald (1994), "Unhappiness and Unemployment”, Economic Journal, 104(424): 648-59.

Dehejia, R.H. and S. Wahba (1999), "Causal effects in nonexperimental studies: Reevaluating the evaluation of training programs", Journal of the American Statistical Association, 94(448): 1053-1062."

Di Tella, R., R. MacCulloch and A.J. Oswald (2003), "The macroeconomics of happiness", Review of Economics and Statistics, 85(4): 809-827.

European Commission, (2001) "European competitiveness report 2001", Luxembourg.

Gilks, W.R, S. Richardson and D.J. Spiegelhalter (eds.) (1996), Markov Chain Monte Carlo in Practice. London: Chapman and Hall.

Heckman, J.J. (1979), "Sample Selection Bias as a Specification Error", Econometrica, 47(1): 153-61.

Heckman, J.J, H. Ichimura and P. Todd (1997), "Matching as an econometric evaluation estimator: Evidence from evaluating a job training programme," Review of Economic Studies, 64: 605-654.

Jaumotte, F. (2003), "Female labour force participation: Past trends and main determinants in OECD countries", Economics Department Working Papers 376, OECD. Paris.

Jonsson, I. (2003) "Policy perspectives on changing intergenerational relations", Social Policy \& Society, 2:3, 241248

Lefèvre, C. and P. Picard (1990), "A non-standard family of polynomials and the final size distribution of Reed-Frost epidemic processes", Advances in Applied Probability, 22: 25-48.

Longini, I.M. and J.S. Koopman (1982), "Household and community transmission parameters from final distributions of infections in households", Biometrics, 38: 115-126.

Nickell, S. (2004), "Poverty and worklessness in Britain", Economic Journal, 114:C1-C25.

OECD(2005), "Economic survey of Spain, 2005", Policy Brief, OECD Observer.

O'Neill, P.D., D.J. Balding, N.G. Becker, M. Eerola and D. Mollison (2000), "Analyses of infectious disease data from household outbreaks by Markov chain Monte Carlo methods", Applied Statistics, 49(4): 517-542.

Poggi, A. (2007a), "Social exclusion mobility in Spain, 1994-2001", Research on Economic Inequality, 14.

Poggi, A. (2007b), “Does persistence of social exclusion exist in Spain?", Journal of Economic Inequality, 5(1): 53-72.

Rosenbaum, P.R. and D.B. Rubin (1983), "The central role of the propensity score in observational studies for casual effects", Biometrika; 70: 41-55. 
Sen A.K. (1985), Commodities and Capabilities, North Holland, Amsterdam. Reprinted in Oxford University Press, Oxford India Paperbacks, 1999.

Sen, A.K. (2000), "Social Exclusion: Concept, Application and Scrutiny," Office of Environment and Social Development, Asian Development Bank, Social Development Papers, 1.

Tsakloglou, P. and F. Papadopoulos (2002), "Identifying population groups at high risk of social exclusion; evidence from the ECHP”, in R.J.A. Muffels, P. Tsakloglou and D.G. Meyes (eds.), Social Exclusion in European Welfare States. Cheltenham; Edward Elgar, Chapter 6.

Whelan, C.T., R. Layte and B. Maitre (2002), "Persistent deprivation in the European Union", Journal of Applied Social Science Studies, 122. 
Table 1. Dimensions of analysis

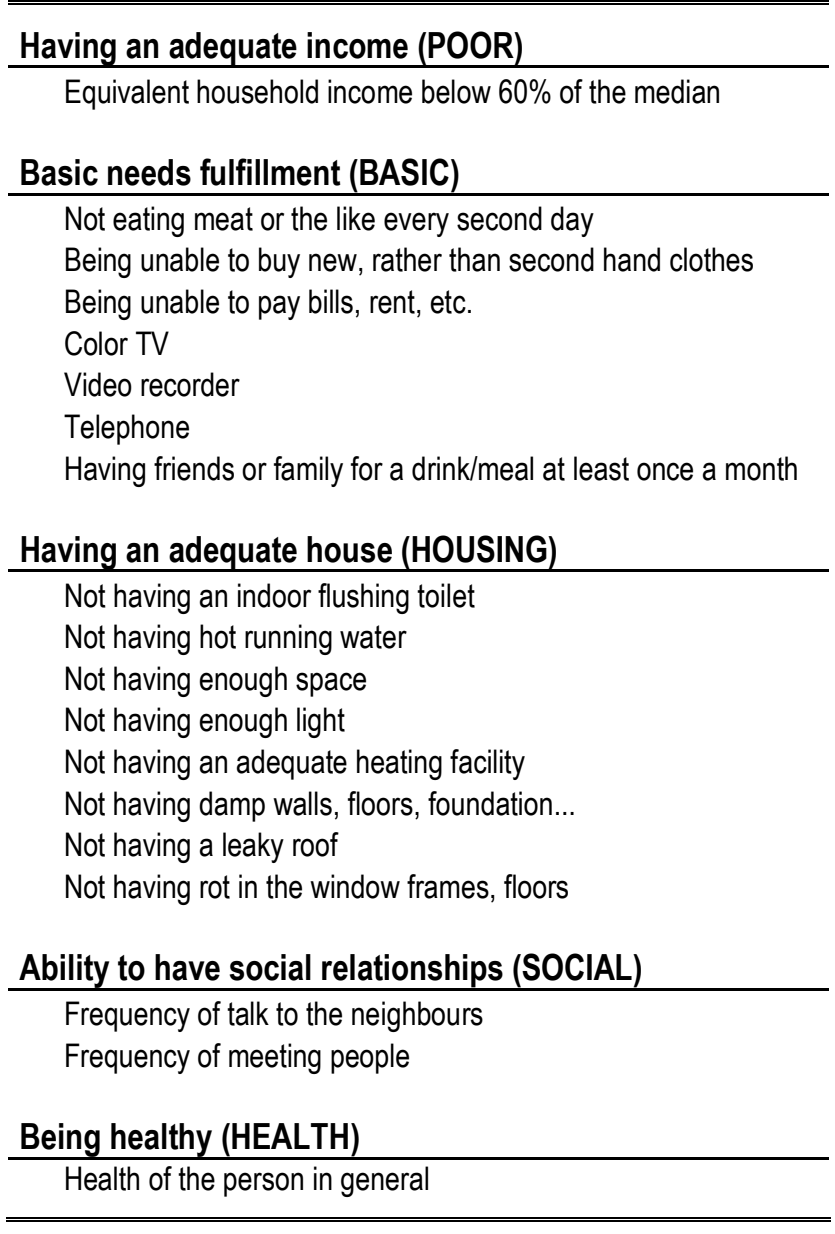

Table 2. Descriptive statistics of treatment groups after matching

\begin{tabular}{|c|c|c|c|c|c|}
\hline \multirow[b]{2}{*}{ Covariate } & \multicolumn{3}{|c|}{ (a) } & \multirow{2}{*}{ (b) } & \multirow{2}{*}{ (c) } \\
\hline & Spain & Italy & All & & \\
\hline Living in consensual union (\%) & 64.94 & 63.76 & 64.39 & 72.93 & 86.63 \\
\hline New member born $(\%)$ & 8.08 & 6.38 & 7.3 & 9.8 & 15.84 \\
\hline One or more children (\%) & 35.37 & 29.36 & 32.43 & 31.7 & 41.99 \\
\hline Low education (\%) & 66.16 & 56.21 & 61.62 & 66.67 & 74.26 \\
\hline Blue collar (\%) & 53.35 & 42.79 & 48.61 & 50.07 & 58.17 \\
\hline Mean monthly wage & 529.14 & 686.76 & 597.93 & 620.24 & 512.43 \\
\hline Mean age & 35.8 & 38.51 & 37.17 & 40.17 & 38.35 \\
\hline \multicolumn{6}{|l|}{ Social exclusion dimensions } \\
\hline Poverty (\%) & 20.27 & 24.5 & 22.52 & 19.46 & 21.04 \\
\hline Basic (\%) & 20.12 & 24.66 & 22.68 & 19.59 & 20.3 \\
\hline Housing (\%) & 25.76 & 13.42 & 20.14 & 17.82 & 19.8 \\
\hline Health $(\%)$ & 5.95 & 4.36 & 5.55 & 6.94 & 4.95 \\
\hline Social (\%) & 7.01 & 13.76 & 10.15 & 9.8 & 7.43 \\
\hline Mean susceptible dimensions & 4.21 & 4.19 & 4.19 & 4.26 & 4.26 \\
\hline No. observations & 657 & 596 & 1261 & 735 & 404 \\
\hline
\end{tabular}

(a) Treatment groups are jobless women in (a); inactive women in (b); inactive women due to housework or child bearing. 
Table 3. Deprivation escape probabilities

Treatment group: jobless women. Control group: women in paid job

\begin{tabular}{|c|c|c|c|c|c|c|}
\hline & \multicolumn{2}{|c|}{ Control group } & \multicolumn{2}{|c|}{ Treatment group } & \multicolumn{2}{|c|}{$\begin{array}{c}\text { Difference: } \\
\text { (treatment-controls) }\end{array}$} \\
\hline & $q_{e}$ & $q_{n}$ & $q_{e}$ & $q_{n}$ & $1-q_{e}$ & $1-q_{n}$ \\
\hline Spain & $\begin{array}{l}0.931 \\
(0.000)\end{array}$ & $\begin{array}{l}0.983 \\
(0.000)\end{array}$ & $\begin{array}{l}0.908 \\
(0.000)\end{array}$ & $\begin{array}{l}0.977 \\
(0.000)\end{array}$ & $2.30 \%$ & $0.60 \%$ \\
\hline Italy & $\begin{array}{l}0.912 \\
(0.003)\end{array}$ & $\begin{array}{l}0.995 \\
(0.004)\end{array}$ & $\begin{array}{l}0.894 \\
(0.000)\end{array}$ & $\begin{array}{l}0.992 \\
(0.000)\end{array}$ & $1.80 \%$ & $0.30 \%$ \\
\hline ALL & $\begin{array}{l}0.939 \\
(0.000)\end{array}$ & $\begin{array}{l}0.987 \\
(0.000)\end{array}$ & $\begin{array}{l}0.901 \\
(0.000)\end{array}$ & $\begin{array}{l}0.984 \\
(0.000)\end{array}$ & $3.80 \%$ & $0.30 \%$ \\
\hline
\end{tabular}

$\overline{\text { Note: standard errors in parenthesis below point estimates }}$

Table 4. Deprivation escape probabilities (pooled sample).

Treatment group: inactive women. Control group: unemployed women

\begin{tabular}{ccc}
\hline \hline \multirow{2}{*}{ Inactive } & $\boldsymbol{q}_{\mathrm{e}}$ & $\boldsymbol{q}_{\boldsymbol{n}}$ \\
\cline { 2 - 3 } & 0.911 & 0.995 \\
Unemployed & $(0.003)$ & $(0.004)$ \\
& 0.885 & 0.944 \\
& $(0.000)$ & $(0.000)$ \\
\hline No. Observations & \multicolumn{2}{c}{735} \\
\hline \hline Note: standard errors in parenthesis below point estimates
\end{tabular}

Table 5. Inactive women: reasons for not looking for a job (pooled sample before matching)

\begin{tabular}{lr}
\hline \hline Reasons for not looking for a job: & Percent \\
\hline Engaged in other activity (study, training, community or military service) & 9.7 \\
Housework, looking after children or other persons & 55.6 \\
Retirement & 17.9 \\
Own illness, injury, incapacitation & 5.0 \\
Other reasons & 11.9 \\
\hline No. Observations & 756 \\
\hline \hline
\end{tabular}

Table 6. Deprivation escape probabilities (pooled sample).

Treatment group: inactive women because housework and child bearing.

Control group: unemployed women

\begin{tabular}{ccc}
\hline \hline & $\boldsymbol{q}_{\mathrm{e}}$ & $\boldsymbol{q}_{\boldsymbol{n}}$ \\
\cline { 2 - 3 } Inactive $\left.{ }^{*}\right)$ & 0.908 & 0.997 \\
& $(0.003)$ & $(0.004)$ \\
Unemployed & 0.916 & 0.928 \\
& $(0.000)$ & $(0.001)$ \\
\hline No. Observations & \multicolumn{2}{c}{404} \\
\hline \hline$\left.{ }^{*}\right)$ because of housework duties and child bearing \\
Note: standard errors in parenthesis below point estimates
\end{tabular}




\section{Appendix A: Evaluation of the occurrence of joblessness}

Matching quality: tests for mean differences before and after matching

\begin{tabular}{|l|cl|cc|cc|}
\hline \multirow{2}{*}{\multicolumn{1}{c|}{ Ho: $\begin{array}{c}\text { Mean }(\text { diff })=0 \\
\operatorname{Pr}(|\mathrm{T}|>|\mathrm{t}|)\end{array}$}} & \multicolumn{2}{c|}{ Spain } & \multicolumn{2}{c|}{ Italy } & \multicolumn{2}{c|}{ All } \\
\cline { 2 - 7 } & before & after & before & after & before & after \\
\hline Living in consensual union & 0.0013 & 0.9999 & 0.1519 & 0.6268 & 0.3245 & 0.5067 \\
New member born & 0.0000 & 0.1357 & 0.0080 & 0.5292 & 0.0000 & 0.9384 \\
No. children & 0.4029 & 0.2534 & 0.0157 & 0.8356 & 0.3354 & 0.2648 \\
Education & 0.0000 & 0.4172 & 0.0000 & 0.3560 & 0.0000 & 0.3715 \\
Blue collar & 0.0000 & 0.0727 & 0.0000 & 0.8642 & 0.0000 & 0.7194 \\
Monthly wage & 0.0000 & 0.0317 & 0.0000 & 0.0184 & 0.0000 & 0.0098 \\
Country & -- & --- & --- & --- & 0.0000 & 0.8928 \\
South & 0.0000 & 0.5917 & 0.0000 & 0.2466 & -- & -- \\
Age & 0.6407 & 0.1701 & 0.0091 & 0.0373 & 0.0805 & 0.1995 \\
\hline Poverty & 0.0000 & 0.9462 & 0.0000 & 0.7754 & 0.0000 & 0.0567 \\
Basic & 0.0000 & 0.5866 & 0.0000 & 0.1911 & 0.0000 & 0.1692 \\
Housing & 0.0000 & 0.6577 & 0.0655 & 0.2936 & 0.0000 & 0.1981 \\
Health & 0.0000 & 0.7327 & 0.0067 & 0.0848 & 0.0000 & 0.6123 \\
Social & 0.7596 & 0.6620 & 0.1737 & 0.5025 & 0.4560 & 0.9478 \\
Susceptible dimensions & 0.0000 & 0.5223 & 0.0000 & 0.0606 & 0.0000 & 0.2873 \\
\hline No. observations & 669 & 657 & 602 & 596 & 1271 & 1261 \\
\hline
\end{tabular}

Logit estimates for the propensity score (calliper $=0.01$ )

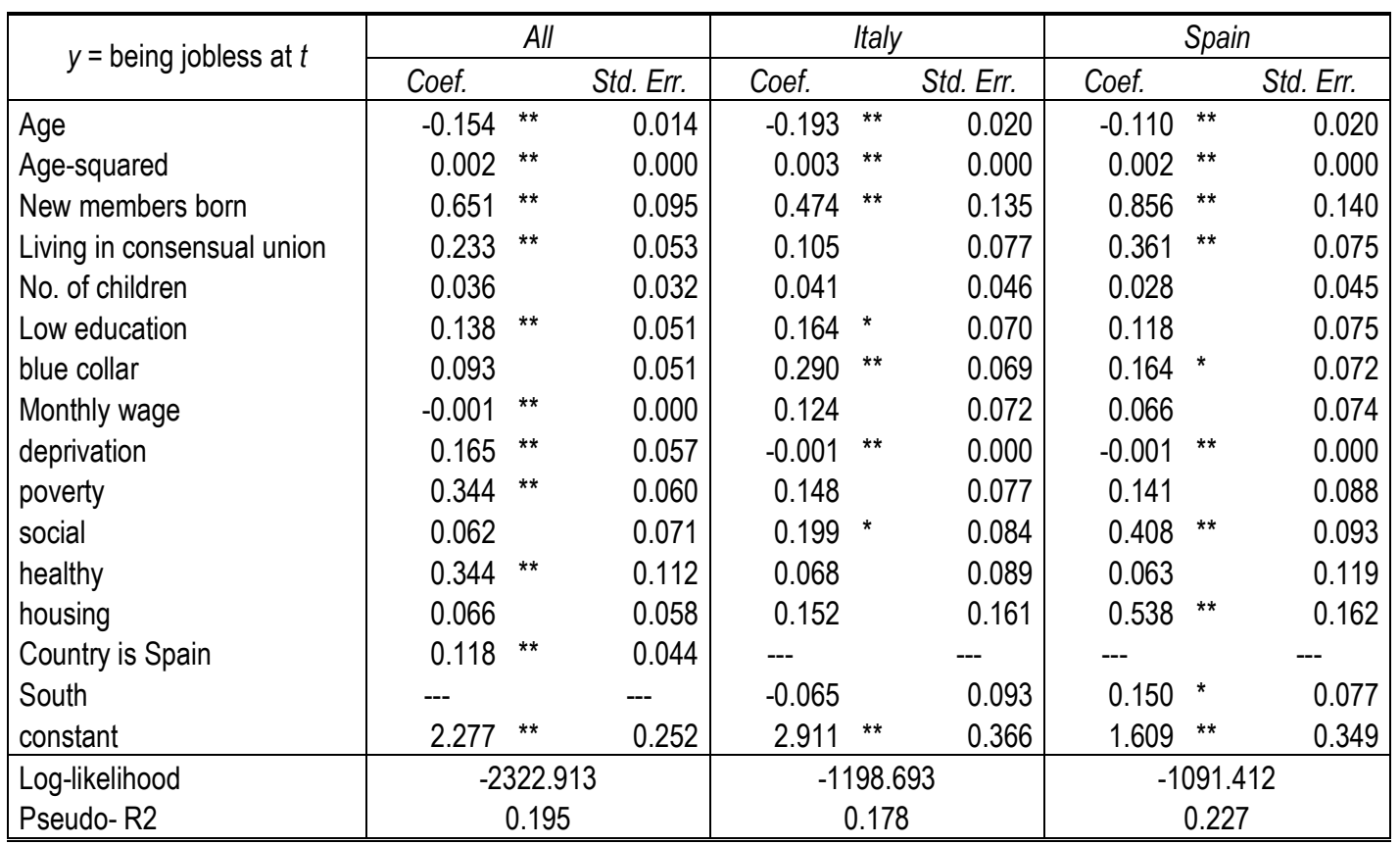

Note: all covariates are measured at $t-1$ 


\section{Appendix B: evaluation of the decision of being inactive}

Matching quality: tests for mean differences before and after matching

\begin{tabular}{lcc}
\hline \multicolumn{1}{c}{$\begin{array}{c}\text { Ho: } \operatorname{mean}(\text { diff })=0 \\
\operatorname{Pr}(|\mathrm{T}|>|\mathrm{t}|)\end{array}$} & Before & \multicolumn{1}{c}{ After } \\
\hline Living in consensual union & 0.000 & 0.385 \\
New member born & 0.000 & 0.795 \\
No. children & 0.027 & 0.306 \\
Education & 0.000 & 0.683 \\
Blue collar & 0.233 & 0.690 \\
monthly wage & 0.005 & 0.095 \\
country & 0.012 & 0.463 \\
age & 0.000 & 0.307 \\
poverty & 0.000 & 0.097 \\
basic & 0.000 & 0.849 \\
housing & 0.023 & 0.228 \\
health & 0.000 & 0.151 \\
social & 0.371 & 0.092 \\
susceptible dimensions & 0.000 & 0.9359 \\
\hline No. Observations & 756 & 735 \\
\hline \hline
\end{tabular}

Logit estimates for the propensity score (calliper $=0.01$ )

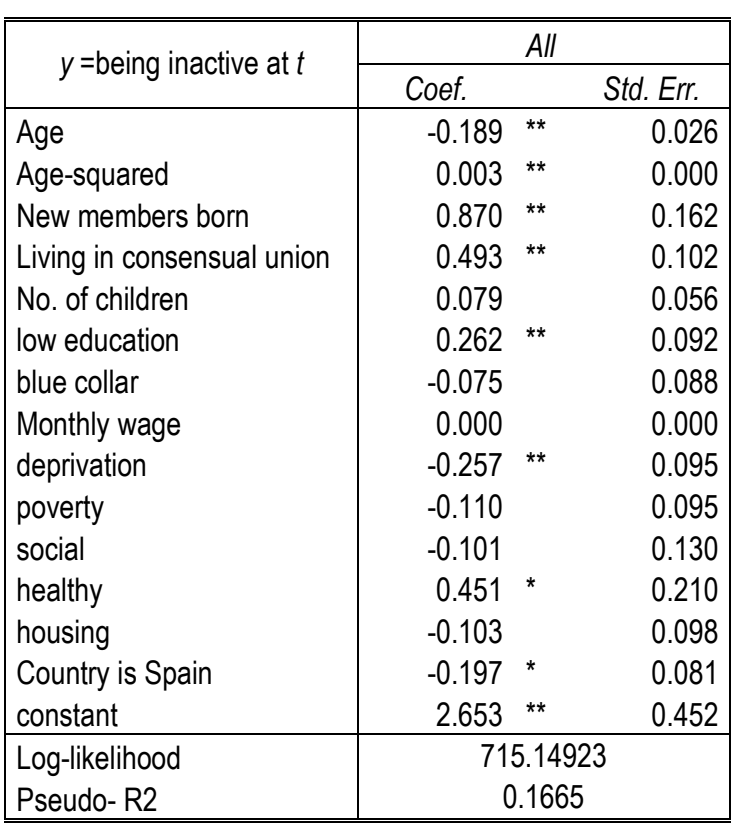

Note: all covariates are measured at $t-1$ 
Appendix C: Evaluation of the decision of being inactive because of housework and child bearing

Matching quality: tests for mean differences before and after matching

\begin{tabular}{lrr}
\hline \multicolumn{1}{c}{$\begin{array}{c}\text { Ho: } \operatorname{mean}(\text { diff })=0 \\
\operatorname{Pr}(|\mathrm{T}|>|\mathrm{t}|)\end{array}$} & \multicolumn{1}{c}{ Before } & \multicolumn{1}{c}{ After } \\
\hline Living in consensual union & 0.000 & 0.052 \\
New member born & 0.000 & 0.852 \\
No. children & 0.447 & 0.618 \\
Education & 0.000 & 0.156 \\
Blue collar & 0.000 & 0.530 \\
Monthly wage & 0.039 & 0.061 \\
Country & 0.269 & 0.692 \\
Age & 0.000 & 0.723 \\
Poverty & 0.011 & 1.000 \\
Basic & 0.004 & 0.608 \\
Housing & 0.310 & 0.538 \\
Health & 0.005 & 0.870 \\
Social & 0.055 & 0.681 \\
Susceptible dimensions & 0.002 & 0.9020 \\
\hline No. Observations & 420 & 404 \\
\hline \hline
\end{tabular}

Logit estimates for the propensity score (calliper=0.01)

\begin{tabular}{|c|c|c|c|}
\hline \multirow{2}{*}{$\begin{array}{l}y=\text { being inactive at } t \text { because } \\
\text { of housework and child bearing }\end{array}$} & \multicolumn{3}{|c|}{ All } \\
\hline & Coef. & & Std. Err. \\
\hline Age & -0.087 & * & 0.033 \\
\hline Age-squared & 0.001 & ** & 0.000 \\
\hline New members born & 1.229 & ** & 0.171 \\
\hline Living in consensual union & 0.919 & ** & 0.124 \\
\hline No. of children & 0.080 & & 0.064 \\
\hline Low education & 0.475 & ** & 0.112 \\
\hline Blue collar & -0.061 & & 0.105 \\
\hline Monthly wage & 0.000 & * & 0.000 \\
\hline Deprivation & -0.221 & * & 0.112 \\
\hline Poverty & -0.120 & & 0.113 \\
\hline Social & -0.214 & & 0.166 \\
\hline Healthy & 0.349 & & 0.251 \\
\hline Housing & -0.078 & & 0.115 \\
\hline Country is Spain & -0.130 & & 0.096 \\
\hline Constant & 0.192 & & 0.577 \\
\hline Log-likelihood & \multirow{2}{*}{\multicolumn{3}{|c|}{$\begin{array}{c}-507.282 \\
0.2114\end{array}$}} \\
\hline Pseudo- R2 & & & \\
\hline
\end{tabular}

Note: all covariates are measured at $t-1$ 